\title{
Modelo basado en Análisis Envolvente de Datos (DEA) para medir la competitividad de las manufactureras peruanas del sector textil
}

\section{Model based on Data Envelopment Analysis (DEA) to measure the competitiveness of Peruvian textile manufacturers}

\author{
Giseth Gamarra-Alván*, Jorge Díaz-Muñante \\ Facultad de Ingeniería de Sistemas e Informática, Universidad Nacional Mayor de San Marcos, Lima, Perú \\ Email Giseth Gamarra-Alván: ggamarraa@unmsmedu.pe \\ Email Jorge Díaz-Muñante: jdiazm@unmsm.edu.pe \\ * Autor para correspondnecia
}

\section{Resumen}

La competitividad proporciona mayores niveles de productividad, eficiencia y calidad, de ahí su importancia para medirla. Este trabajo presenta un modelo DEA para medir la competitividad de las manufactureras del sector textil peruano. El modelo propuesto consta de dos fases: la primera fase, mide los niveles de desarrollo de la calidad, innovación y eficiencia de las empresas; la segunda fase la competitividad de las empresas considerando su calidad innovación y eficiencia. Para ilustrar el modelo propuesto se usó el software SIAD aplicado a 16 empresas del sector de manufactura textil, y se muestra el ranking de las empresas por cada factor y por la competitividad.

Palabras clave: Competitividad; Análisis Envolvente de Datos; Industria textil.

\begin{abstract}
Competitiveness provides higher levels of productivity, efficiency and quality, hence its importance to measure it. This paper presents a DEA model to measure the competitiveness of Peruvian textile sector manufacturers. The proposed model consists of two phases: the first phase, measures the levels of development of quality, innovation and efficiency of the companies; the second phase the competitiveness of companies considering their quality innovation and efficiency. To illustrate the proposed model, the SIAD software applied to 16 companies in the textile manufacturing sector was used, and the ranking of companies by each factor and competitiveness is shown.
\end{abstract}

Keywords: Competitiveness; Data Envelopment Analysis; Textile Industry.

Gamarra G, Díaz J. Modelo basado en Análisis Envolvente de Datos (DEA) para medir la competitividad de las manufactureras peruanas del sector textil. Revista Peruana de Computación y Sistemas 2018 1(1):35-46. http://dx.doi.org/10.15381/xxxxxxs

(C) Los autores. Este artículo es publicado por la Revista Peruana de Computación y Sistemas de la Facultad de Ingeniería de Sistemas e Informáticade la Universidad Nacional Mayor de San Marcos. Este es un artículo de acceso abierto, distribuido bajo los términos de la licencia Creative Commons Atribucion - No Comercia_Compartir Igual 4.0 Internacional. (http://creativecommons.org/licenses/by-nc-sa/4.0/) que permite el uso no comercial, distribución y reproducción en cualquier medio, siempre que la obra original sea debidamente citada. 


\section{Introdución}

En su lucha por conseguir un alto nivel de rendimiento, las economías mundiales han convertido en un objetivo el hecho de alcanzar la competitividad internacional. Sin embargo, para concebir la competitividad internacional del país, es imprescindible evaluar la competitividad de las empresas, ya que la economía del país se basa primordialmente en las actividades económicas que realizan las empresas. Es preciso aclarar que la competitividad también puede verse afectada por factores externos como el entorno macroeconómico y la estabilidad a nivel nacional, dado que son factores no ajenos que desempeñan un papel importante.

El término "competitivo" es muy utilizado, pero, ¿qué es ser competitivo? [1] define un sector muy competitivo si su balanza comercial es positiva y si la cuota de exportación de la industria supera el promedio nacional. Años siguientes, [2] se refiere a un sector muy competitivo si mantiene una cuota de mercado estable o en crecimiento y las ganancias son satisfactorias para todas las empresas de la industria. Por otro lado, [3] considera que la productividad del trabajo, la integración vertical, la innovación tecnológica y el tamaño de la empresa son los factores críticos para la competitividad.

En la investigación realizada por [4], se indica que el mercado competitivo y la competitividad empresarial son la calidad global de una empresa para seguir proporcionando productos o servicios al mercado con más eficacia que otras empresas a fin de obtener beneficios y su propio desarrollo.

El sector textil peruano genera entre 350 y $400 \mathrm{mil}$ empleos directos, además, representa el 1.9\% del PBI y poco más del $10 \%$ del de la manufactura. Sin embargo, las exportaciones del sector textil confecciones representaban el $17 \%$ del total de exportaciones no tradicionales con 1,918 millones de dólares, aun cuando esa cifra fue $-11-9 \%$ respecto a 2012, pero en 2016 representó 1196 millones de dólares con una variación de $-10.2 \%$ con respecto a 2015 [5].

En la literatura especializada existen diversos modelos para medir la competitividad de las empresas, algunas se basan en modelos [6], [7], [8], [9], fórmulas [10] y marcos [11]; sin embargo, no hay un modelo estándar. Los factores son medidos en forma cualitativa mediante juicio de experto, lo cual no es tan preciso. DEA se encuentra directamente relacionada con las variables eficiencia, calidad e innovación que, según Mauricio [12], son factores internos que definen el grado de competitividad en la industria y servicios. Todos los aportes existentes establecen un ranking, pero la DEA permite, además, un análisis de factores por medio de la frontera eficiente que se determina.

Por lo antes expuesto, en este trabajo proponemos un modelo de análisis envolvente de datos para medir la competitividad de las manufactureras peruanas del sector textil aplicando Análisis Envolvente de Datos (DEA). Este artículo se organiza en cinco secciones.
En la sección dos hacemos una revisión de la literatura sobre el concepto de competitividad, como se define en diversos escenarios. En la sección tres se presenta el modelo propuesto. Los resultados del modelo propuesto aplicado a 16 empresas manufactureras peruanas se presentan en la sección cuatro. Finalmente, las conclusiones se encuentran en la sección cinco.

\section{Revisión de literatura}

En esta sección se detalla la revisión de la literatura con respecto a la evaluación de la competitividad, la cual está dividida en tres secciones: en la primera sección está la definición de competitividad, en la segunda sección se encuentran las variables de la competitividad, y en la tercera sección se hallan los métodos revisados.

En la revisión de la literatura se han encontrado términos como Determinantes, Dimensiones, Indicadores y Factores, para hacer referencia a la característica de nivel superior (dentro de un método) que agrupa una diversidad de variables.

\subsection{Competitividad}

Se define como una condición que permite que un país o una empresa opere de manera más eficiente o desde otra perspectiva con mayor calidad que sus competidores, y de este modo resulte en beneficios acumulados [13].

La competitividad de un país es definida por Potter [1] con el modelo Diamante, en el que destaca las dimensiones de producción, como la mano de obra calificada e infraestructura y las dimensiones críticas de la nación para competir en una industria determinada. Pero hay quienes lo refutan, por ejemplo, [15] indica que Porter solo enfoca la competitividad en términos de exportación. [16] propone el modelo Nine-Factor que se clasifica en cuatro categorías: la sujeción, el medio ambiente, los recursos y los mecanismos, donde se abarca tanto los recursos físicos como los humanos. [17] crea un modelo ampliado, donde incorpora la innovación tecnológica y la difusión, las actividades comerciales internacionales y el papel del gobierno en los modelos anteriores para medir la competitividad del país. [18] predicen la competitividad de los países mediante el análisis de cuatro métodos de descubrimiento de conocimiento: a) los modelos de regresión por pasos, b) modelos de programación lineal ponderada, c) redes neuronales, y d) los árboles de clasificación y regresión. Desde 2005, el Foro Económico Mundial ha publicado informes sobre la competitividad mundial de más de 100 países que sirven como puntos de referencia para los responsables políticos y otras partes interesadas para juzgar el éxito competitivo de sus países en un contexto mundial [19].

Un primer intento de cuantificar la competitividad industrial se basó en la competitividad de un fabricante considerando su dominio industrial, el costo de superioridad, y el ambiente político-económico [20]. [21] afirman que la exploración de las relaciones cuantitativas 
entre la capacidad de innovación tecnológica y la competitividad sugiere una estrecha relación interna entre estas dos variables. [22], desde el sector del automóvil de Polonia, sugieren que la transferencia de conocimientos de las empresas transnacionales mejora el rendimiento de los proveedores locales $y$, posteriormente, aumenta su capacidad para competir.

En cuanto a la competitividad a nivel de empresas, [10] indican que las empresas deben ser operativamente eficientes, rentables y conscientes de la calidad para ofrecer a los clientes un mayor valor y satisfacción con respecto a los competidores. [6] recalca que la competitividad implica una combinación de activos y procesos, donde se heredan los activos (recursos naturales) o son creados (infraestructura), y los procesos transforman los activos para lograr beneficios económicos de las ventas a clientes. [7] precisa que una empresa tiene ventajas competitivas si se puede producir y vender en los mercados competitivos productos homogéneos por precio más bajo que sus rivales sin subsidios, o si se puede desarrollar características únicas para los productos disponibles y los productos innovadores. Finalmente, para [8], la empresa debe mantenerse al día con el ritmo del mercado y tratar de funcionar lo más eficiente posible. Si no lo hace, no será capaz de mantener su posición, ni obtener beneficios suficientes y, finalmente, dejará de existir.

\subsection{Variables}

De la revisión de la literatura, se han identificado 44 variables que se usan para medir la competitividad de las empresas del sector manufactura textil y que se muestran en la Tabla 1.

\subsection{Métodos para medir competitividad}

A continuación, se revisa los métodos que han sido propuestos para medir la competitividad en las organizaciones de diferentes sectores y del sector textil.

\subsubsection{Método de Clasificación de experto.-}

[23] analiza de manera cuantitativa y cualitativa cada uno de los aspectos que se deben tener en cuenta para la evaluación de la competitividad de las empresas de tecnología. Los pesos indicados son adoptados del método de clasificación de expertos. Se aplica el método integral difuso, que se utiliza para la evaluación de la competitividad empresarial de alta tecnología, se ocupa de los problemas que son difusos y difíciles de cuantificar, ayuda a analizar la situación actual de la competitividad de la empresa y permite mejorar su estrategia tecnológicamente competitiva para mejorar su competitividad. Sin embargo, el método de clasificación de expertos que determina el peso de cada índice entrega valores subjetivos a la investigación, por lo que los resultados no pueden determinarse como objetivos; otros métodos tampoco resuelven este problema fundamental, por lo que todavía hay que explorar más. El método aplicado es, en general, muy claro y específico, sin embargo, sus resultados son subjetivos.
Tabla 1: Variables para medir competitividad del sector textil

\begin{tabular}{|c|c|}
\hline Variables & Referencia \\
\hline $\begin{array}{l}\text { Calidad del producto } \\
\text { Salario } \\
\text { Buenas condiciones de trabajo / Cuidado de la Salud } \\
\text { Formación } \\
\text { Velocidad al mercado } \\
\text { Productividad de los trabajadores } \\
\text { Buenas condiciones de trabajo / Salud } \\
\text { Relación previa de largo plazo con su comprador } \\
\text { Salarios dignos de los trabajadores } \\
\text { Avances tecnológicos } \\
\text { Asistencia / capacidad de pre-producción para mane- } \\
\text { jar diseños / materiales sofisticados } \\
\text { Formación de los trabajadores } \\
\text { Bajo costo }\end{array}$ & [8] \\
\hline Disponibilidad, calidad y accesibilidad & \multirow{13}{*}{ [9] } \\
\hline $\begin{array}{l}\text { Calidad de primaria, secundaria y preparatoria, edu- } \\
\text { cación superior y capacitación en el trabajo, producti- }\end{array}$ & \\
\hline $\begin{array}{l}\text { Presencia de I + D, cooperación de universidades con } \\
\text { la industria y nivel de investigación aplicada, presen- } \\
\text { cia de investigación nacional y fondos }\end{array}$ & \\
\hline $\begin{array}{l}\text { Calidad del mercado de capitales, accesibilidad a } \\
\text { las finanzas y oportunidad de inversión extranjera, } \\
\text { retorno relativo de la inversión }\end{array}$ & \\
\hline $\begin{array}{l}\text { Calidad de la infraestructura básica (carreteras, puer- } \\
\text { tos eléctricos) e infraestructura avanzada (telecomu- } \\
\text { nicaciones, logística) } \\
\text { Disponibilidad de información comercial y de merca- } \\
\text { do, uso de e.C } \\
\text { Calidad y costo de administración y logística, ambien- } \\
\text { te regulatorio }\end{array}$ & \\
\hline $\begin{array}{l}\text { Accesibilidad del núcleo y tecnología de apoyo, posi- } \\
\text { bilidad de difusión de tecnología, innovación }\end{array}$ & \\
\hline $\begin{array}{l}\text { Capital de mercado, apertura de contratos del sector } \\
\text { público. } \\
\text { El grado de sofisticación del comprador, la calidad de } \\
\text { la demanda y el estándar de la regulación, la tasa de } \\
\text { cambio de la necesidad del cliente, la posibilidad de } \\
\text { reemplazo del producto }\end{array}$ & \\
\hline $\begin{array}{l}\text { Cuota de mercado, cuota de mercado exterior y } \\
\text { segmento de mercado }\end{array}$ & \\
\hline $\begin{array}{l}\text { Calidad y cantidad de industrias relacionadas, calidad } \\
\text { del proveedor }\end{array}$ & \\
\hline Calidad y cantidad de las industrias de apoyo & \\
\hline $\begin{array}{l}\text { Intensidad competitiva, presencia de barreras de } \\
\text { entrada, diferenciación }\end{array}$ & \\
\hline $\begin{array}{l}\text { Condiciones comerciales, política laboral, incertidum- } \\
\text { bre económica y política }\end{array}$ & \\
\hline $\begin{array}{l}\text { Enfoque estratégico, cadena de valor, prácticas de } \\
\text { gestión y metas de los individuos } \\
\text { Nivel de tasa de interés, nivel de inflación, estabilidad } \\
\text { del tipo de cambio } \\
\text { Apoyo gubernamental, apoyo a las exportaciones, } \\
\text { apoyo gubernamental a la tecnología, capacitación, } \\
\text { rigurosidad del reglamento }\end{array}$ & \\
\hline $\begin{array}{l}\text { Recursos físicos } \\
\text { Recursos humanos } \\
\text { Recursos de información } \\
\text { Recursos de capital } \\
\text { Necesidades del consumidor } \\
\text { Sofisticación de la demanda } \\
\text { Infraestructura } \\
\text { Industrias relacionadas } \\
\text { Incentivos de inversión } \\
\text { Factores competitivos } \\
\text { Instituciones políticas y económicas } \\
\text { Subvenciones del Estado } \\
\text { Controles estatales }\end{array}$ & [6] \\
\hline
\end{tabular}




\subsubsection{Método de Redes bayesianas.-}

[24] propone un marco en el que se analizan las diferentes variables de la competitividad. Para realizar el análisis de la competitividad, el autor establece el uso de una metodología que define en un marco con 3 etapas, las cuales son detalladas en la Fig. 1, y el uso de las redes bayesianas causales (BCNs).

La primera etapa es la estructuración del problema. Los indicadores del WEF son las entradas y una lista de indicadores relacionados con la industria del automóvil son los resultados. Con ello se crea una lista como resultado de una encuesta en línea de los miembros de los grupos de interés de la industria automotriz.

La segunda etapa de modelado causal toma la salida de la Etapa 1 como entrada y genera la BCN (Redes bayesianas causales) de la industria del automóvil como la salida. En un principio, las relaciones entre los indicadores se determinan a través de un taller en el que los participantes son skateholders de la industria de automovilística. En este taller, los expertos evalúan las relaciones causales entre los indicadores de competitividad de la industria automotriz. Posteriormente, se desarrolla un BCN mediante la integración de los resultados del taller con los datos del Foro Económico Mundial.

En la etapa final, se utiliza el BCN para analizar la competitividad de la industria automovilística de Turquía sobre la base de datos de los indicadores relacionados con la industria automovilística en diferentes escenarios. Las salidas de la tercera etapa son las sugerencias de políticas que se deben desarrollar con el fin de dar forma al futuro de la competitividad de la industria automotriz.

El uso de BCNs en la metodología hace posible decodificar las relaciones entre las variables de interés y el modelo de las relaciones causales y, de este modo, contribuir al entendimiento de las dimensiones básicas que son relevantes para la competitividad de la industria automotriz. En concreto, el BCN permite un análisis en de las relaciones causales entre las variables y hace posible probar diferentes escenarios que incorporan intervenciones políticas y la incertidumbre del futuro. La evidencia para el uso exitoso de la BCN de tal dominio es relevante e importante para la disciplina de la OR.

\begin{tabular}{|l|l|l|l|}
\hline $\begin{array}{l}\text { Nivel 1: } \\
\text { Problema de }\end{array}$ & $\begin{array}{l}\text { Indicadores } \\
\text { WEF }\end{array}$ & $\begin{array}{l}\text { Experto evalúa } \\
\text { Indicadores } \\
\text { WEF }\end{array}$ & $\begin{array}{l}\text { Indicadores } \\
\text { con relación } \\
\text { automotriz }\end{array}$ \\
\hline \begin{tabular}{l|l|l|} 
Nivel 2: \\
Modelamientc \\
casual
\end{tabular} & $\begin{array}{l}\text { Indicadores } \\
\text { con relación } \\
\text { automotriz }\end{array}$ & $\begin{array}{l}\text { Experto evalúa } \\
\text { relación casual }\end{array}$ & $\begin{array}{l}\text { BCN de } \\
\text { industria } \\
\text { automotriz }\end{array}$ \\
\hline $\begin{array}{l}\text { Nivel 3: } \\
\text { Análisis }\end{array}$ & $\begin{array}{l}\text { BCN de } \\
\text { industria } \\
\text { automotriz }\end{array}$ & $\begin{array}{l}\text { Análisis de BCN } \\
\text { bajo escenarios }\end{array}$ & $\begin{array}{l}\text { Sugerencia } \\
\text { de política }\end{array}$ \\
\hline
\end{tabular}

Fig. 1: Marco para la evaluación de la competitividad en la industria automovilística Turca [24]
La estructura de un BCN es más transparente que los métodos de caja negra, como el RNA, y esta transparencia permite una comunicación eficiente e interactiva. Esta combinación de datos objetivos y juicios subjetivos de expertos permite BCNs para producir modelos coherentes de los sistemas de investigación.

La estructura de un BCN es más transparente que los métodos de caja negra, como el RNA, y esta transparencia permite una comunicación eficiente e interactiva. Esta combinación de datos objetivos y juicios subjetivos de expertos permite BCNs para producir modelos coherentes de los sistemas de investigación.

\subsubsection{Método Diamante de Porter y Método del indice.-}

[25] explica que para medir la competitividad se apoya en la aplicación de dos aportes: por un lado, para medir la competitividad de manera cualitativa, usa el conocido diamante de Porter (Tabla 2), mientras que para medir la competitividad de manera cuantitativa aplica la fórmula de [26].

En definitiva, la competitividad se refiere a los arreglos institucionales y de políticas que crean las condiciones en las que la productividad puede crecer sostenible (crecimiento de la productividad es la única fuente de crecimiento sostenido de los ingresos, a su vez, la columna vertebral de los niveles de vida crecientes).

Las perspectivas sobre la competitividad de las exportaciones rumanas podrá ser prometedor si: (1) la competitividad internacional de las exportaciones rumanas se sustentan en: (a) la reducción de los costos de producción al mismo tiempo que en la mejora de la calidad de los productos, los cuales son decisivos, (b) estimular y ayudar a las empresas exportadoras para penetrar nuevos mercados, (c) el mantenimiento de la tasa de cambio a un nivel razonable [27]; (2) o si se mantiene el déficit por cuenta corriente a un nivel sostenible.

Tabla 2: Aporte de Mitschke sobre el diamante de Porter para medir la competitividad de un país [25]

\begin{tabular}{|c|c|c|c|}
\hline & \multicolumn{2}{|c|}{$\begin{array}{l}\text { Dos dimensiones de la competitividad internacional de } \\
\text { naciones }\end{array}$} \\
\hline & & $\begin{array}{l}\text { Atractivos locales de } \\
\text { naciones }\end{array}$ & $\begin{array}{l}\text { Competitividad internacional de } \\
\text { empresas locales }\end{array}$ \\
\hline \multirow{3}{*}{ 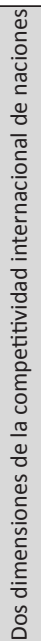 } & Gobierno & \multicolumn{2}{|c|}{$\begin{array}{c}\text { Política fiscal / Política de impuesto } \\
\text { Política monetaria: baja inflación y baja tasa de interés. } \\
\text { Política monetaria. } \\
\text { Regulación de capital de mercado } \\
\text { Infraestructura publica } \\
\text { Estándar social y ecológico } \\
\text { Política comercial y política de competencia: abierto y libre } \\
\text { mercado } \\
\text { Política de investigación y desarrollo }\end{array}$} \\
\hline & & $\begin{array}{l}\text { Zonas económicas } \\
\text { especiales para inver- } \\
\text { sores extranjeros }\end{array}$ & $\begin{array}{c}\text { Política industrial para las empresas } \\
\text { nacionales, la política de I + D } \\
\text { fomenta la asunción de riesgos y la } \\
\text { laboriosidad } \\
\end{array}$ \\
\hline & $\begin{array}{l}\text { Empresas } \\
\text { locales }\end{array}$ & $\begin{array}{l}\text { Cluster industrial } \\
\text { competencia } \\
\text { pro-competitiva } \\
\text { disposición para la } \\
\text { cooperación en I + D } \\
\text { servicios bancarios } \\
\text { atractivos }\end{array}$ & $\begin{array}{l}\text { Innovación pro-competitiva. } \\
\text { Productividad del comportamiento. } \\
\text { Estrategia de negocio de inter- } \\
\text { nacionalización. Capacidades de } \\
\text { gestión de las empresas. Orientación } \\
\text { específica para la exportación de } \\
\text { capital humano }\end{array}$ \\
\hline
\end{tabular}




\subsubsection{Método de Caras Chernooff.-}

[28] indica que para la evaluación de la competitividad mediante el modelo de caras de Chernoff, cada una de las características faciales es representativa de una dimensión de datos particular. La primera dimensión de datos se puede asociar con el tamaño de la boca, la segunda es el tamańo de la nariz, la tercera son los ojos, etc. Esta técnica de datos altamente concentrados refleja las características de la tabla de datos a través de las imágenes. Con base a este estudio, se puede obtener la posición final de competitividad de los diez puertos en evaluación.

A continuación, se presenta una breve descripción de cada uno de los indicadores evaluados:

a. El tamaño y la forma de la cara: representan, respectivamente, el medio natural y el desarrollo económico entorno del puerto.

b. La longitud de la nariz: significa la capacidad de atraques del puerto.

c. La posición de la boca: significa el equipo de manipulación portuaria.

d. Boca flexión: significa la capacidad de almacenamiento.

e. Ancho de boca: significa eficiencia de la operación.

f. Ojos posición: significa la eficiencia del despacho de aduana.

g. El espacio entre los ojos: significa el tiempo medio de parada en el puerto.

h. Los puntos de la vista: significa un medio de cobertura de rutas.

i. La forma del ojo: significa condiciones de recojo y distribución.

j. El ancho de los ojos: significa nivel del tipo de operación.

k. Posición del globo del ojo: significa el sistema EDI.
1. Posición de cejas: significa el sistema de monitoreo de seguridad.

m. Cejas ángulo: significa las ganancias por acción.

n. El ancho de las cejas: significa la tasa de rendimiento de carga de casi cinco años.

\subsubsection{Método del Índice.-}

[6], para la medición de la competitividad, desarrolló un índice compuesto de cinco factores (condiciones de los factores, condiciones de la demanda, relacionados y apoyo a las industrias, estrategia de la empresa, la estructura y la rivalidad, y las políticas del gobierno) (ver Tabla 3); se desarrollan los coeficientes de estos factores, los cuales se determinan a través del método de la opinión de expertos.

Las variables que se definieron son las siguientes: recursos físicos, recursos humanos, recursos de información y recursos de capital, para evaluar Condiciones de los factores, Necesidades del consumidor y Sofisticación de la demanda para Condiciones de demanda, Infraestructura e industrias relacionadas para Industrias relacionadas y de apoyo, Incentivos de inversión y Factores competitivos para Estrategia de firma, estructura, rivalidad. Finalmente, Instituciones políticas y económicas, Subvenciones del Estado y Controles estatales para Política gubernamental.

El método Índice, desarrollado con el fin de investigar los niveles de competitividad, indica que el 65,4\% de la competitividad de las empresas se encuentra en un nivel medio, el $17,3 \%$ está por encima de la media y el $17,3 \%$ está por debajo de ella.

Sin embargo, no se aclaran las razones fundamentales detrás de la diferenciación de estos niveles de competitividad. Los pesos de cada índice definido son obtenidos a través del método de experto, lo cual no permite que esta investigación asevere los resultados.

Así también [9] motivado por resolver dos problemáticas, la primera orientada hacia la evaluación de la competitividad basada en indicadores económicos, del cual comenta que el valor de un índice de competitividad depende del marco analítico y la metodología aplicada en la clasificación; la segunda problemática en-

Tabla 3: Identificación de factores aplicado a las manufactureras de la región TRB1 [6]

\begin{tabular}{|c|c|c|c|c|}
\hline $\begin{array}{l}\text { I. Condiciones de los } \\
\text { factores }\end{array}$ & $\begin{array}{l}\text { II. Condiciones de } \\
\text { demanda }\end{array}$ & $\begin{array}{l}\text { III. Industrias relacionadas } \\
\text { y de apoyo }\end{array}$ & $\begin{array}{l}\text { IV. Estrategia de firma, } \\
\text { estructura, rivalidad }\end{array}$ & V. Política gubernamental \\
\hline FC1. Recursos físicos & $\begin{array}{l}\text { DC1. Necesidades del } \\
\text { consumidor }\end{array}$ & RSS1. Infraestructura & $\begin{array}{l}\text { FSSR1. Incentivos } \\
\text { de inversión }\end{array}$ & $\begin{array}{l}\text { GP1. Instituciones políti- } \\
\text { cas y económicas }\end{array}$ \\
\hline FC2. Recursos humanos & $\begin{array}{l}\text { DC2. Sofisticación de la } \\
\text { demanda }\end{array}$ & $\begin{array}{l}\text { RSS2. Industrias relacio- } \\
\text { nadas }\end{array}$ & $\begin{array}{l}\text { FSSR2. Factores compe- } \\
\text { titivos }\end{array}$ & $\begin{array}{l}\text { GP2. Subvenciones del } \\
\text { Estado }\end{array}$ \\
\hline $\begin{array}{l}\text { FC3. Recursos de infor- } \\
\text { mación }\end{array}$ & & & & GP3. Controles estatales \\
\hline FC4. Recursos de capital & & & & \\
\hline
\end{tabular}


caminada a la evaluación de la competitividad basada en factores de Porter, en el que describe el enfoque propuesto por [29], describiéndolo como uno de los más integrales.

[9] propone, entonces, un modelo donde considera cinco determinantes (Tabla 4), cada uno compuesto de dos a seis elementos, y cada elemento incluye un número de variables, es decir, 55 variables en total. Así, afirma que la competitividad de una industria o una empresa se miden con base en la ponderación de los elementos. Cada variable se califica en una escala que va de 0 a 3 . Esto se utiliza como base para el cálculo de la competitividad de cada elemento. Además, la importancia relativa de cada elemento se define dentro de un rango de 1 a 4 .

Tabla 4: Lista de determinantes, elementos y variables que afectan el desempeño competitivo [9]

\begin{tabular}{|c|c|c|}
\hline Determinante & Elemento & Variables \\
\hline \multirow{8}{*}{$\begin{array}{l}\text { Condiciones } \\
\text { de los } \\
\text { factores }\end{array}$} & Materias primas & Disponibilidad, calidad y accesibilidad \\
\hline & $\begin{array}{l}\text { Recursos } \\
\text { humanos }\end{array}$ & $\begin{array}{l}\text { Calidad de primaria, secundaria y } \\
\text { preparatoria, educación superior y ca- } \\
\text { pacitación en el trabajo, productividad } \\
\text { y costo de los recursos humanos }\end{array}$ \\
\hline & $\begin{array}{l}\text { Factor } \\
\text { especializado }\end{array}$ & $\begin{array}{l}\text { Presencia de I + D, cooperación de } \\
\text { universidades con la industria y nivel } \\
\text { de investigación aplicada, presencia de } \\
\text { investigación nacional y fondos }\end{array}$ \\
\hline & Capital & $\begin{array}{l}\text { Calidad del mercado de capitales, acce- } \\
\text { sibilidad a las finanzas y oportunidad de } \\
\text { inversión extranjera, retorno relativo de } \\
\text { la inversión }\end{array}$ \\
\hline & $\begin{array}{l}\text { Infraestructura } \\
\text { física }\end{array}$ & $\begin{array}{l}\text { Calidad de la infraestructura básica } \\
\text { (carreteras, puertos eléctricos) e } \\
\text { infraestructura avanzada (telecomuni- } \\
\text { caciones, logística) }\end{array}$ \\
\hline & $\begin{array}{l}\text { Infraestructura } \\
\text { de información }\end{array}$ & $\begin{array}{l}\text { Disponibilidad de información comer- } \\
\text { cial y de mercado, uso de e.C }\end{array}$ \\
\hline & $\begin{array}{l}\text { Infraestructura } \\
\text { administrativa y } \\
\text { logística }\end{array}$ & $\begin{array}{l}\text { Calidad y costo de administración y } \\
\text { logística, ambiente regulatorio }\end{array}$ \\
\hline & Tecnología & $\begin{array}{l}\text { Accesibilidad del núcleo y tecnología } \\
\text { de apoyo, posibilidad de difusión de } \\
\text { tecnología, innovación }\end{array}$ \\
\hline \multirow{5}{*}{$\begin{array}{l}\text { Condiciones } \\
\text { de la } \\
\text { demanda }\end{array}$} & Mercado local & $\begin{array}{l}\text { Capital de mercado, apertura de contra- } \\
\text { tos del sector público }\end{array}$ \\
\hline & $\begin{array}{l}\text { Calidad de } \\
\text { demanda }\end{array}$ & $\begin{array}{l}\text { El grado de sofisticación del comprador, } \\
\text { la calidad de la demanda y el estándar } \\
\text { de la regulación, la tasa de cambio de la } \\
\text { necesidad del cliente, la posibilidad de } \\
\text { reemplazo del producto }\end{array}$ \\
\hline & $\begin{array}{l}\text { Exportación de } \\
\text { cuota de } \\
\text { mercado }\end{array}$ & $\begin{array}{l}\text { Cuota de mercado, cuota de mercado } \\
\text { exterior y segmento de mercado }\end{array}$ \\
\hline & $\begin{array}{l}\text { Industria } \\
\text { relacionada }\end{array}$ & $\begin{array}{l}\text { Calidad y cantidad de industrias relacio- } \\
\text { nadas, calidad del proveedor }\end{array}$ \\
\hline & $\begin{array}{l}\text { Industrias de } \\
\text { apoyo }\end{array}$ & $\begin{array}{l}\text { Calidad y cantidad de las industrias de } \\
\text { apoyo }\end{array}$ \\
\hline \multirow{3}{*}{$\begin{array}{l}\text { Industrias } \\
\text { relacionadas y } \\
\text { de apoyo }\end{array}$} & $\begin{array}{l}\text { Estructura y } \\
\text { rivalidad }\end{array}$ & $\begin{array}{l}\text { Intensidad competitiva, presencia de } \\
\text { barreras de entrada, diferenciación }\end{array}$ \\
\hline & $\begin{array}{l}\text { Clima de } \\
\text { yinversión }\end{array}$ & $\begin{array}{l}\text { Condiciones comerciales, política labo- } \\
\text { ral, incertidumbre económica y política }\end{array}$ \\
\hline & Estrategia & $\begin{array}{l}\text { Enfoque estratégico, cadena de valor, } \\
\text { prácticas de gestión y metas de los } \\
\text { individuos }\end{array}$ \\
\hline $\begin{array}{l}\text { Estrategia } \\
\text { de firma, } \\
\text { estructura, } \\
\text { rivalidad }\end{array}$ & $\begin{array}{l}\text { Estabilidad ma- } \\
\text { cro económica }\end{array}$ & $\begin{array}{l}\text { Nivel de tasa de interés, nivel de infla- } \\
\text { ción, estabilidad del tipo de cambio }\end{array}$ \\
\hline Gobierno & Ambiente & $\begin{array}{l}\text { Apoyo gubernamental, apoyo a las } \\
\text { exportaciones, apoyo gubernamental a } \\
\text { la tecnología, capacitación, rigurosidad } \\
\text { del reglamento }\end{array}$ \\
\hline
\end{tabular}

En consecuencia, las empresas productoras de materias primas deben mejorar continuamente su ventaja competitiva mediante la mejora de sus procesos y productos. Otro elemento importante de la condición de los factores es el componente de recursos humanos. Una de las limitaciones de los recursos humanos es el limitado acceso al desarrollo de habilidades y de un adecuado entrenamiento en el puesto de trabajo.

\subsubsection{Método Opinión de Experto.-}

[8], en su estudio para comprender mejor los puntos de vista de los principales actores en la industria del vestido y las nociones clave de la productividad y la competitividad, realizaron entrevistas estructuradas, con cuatro grupos de actores: los compradores internacionales, los funcionarios gubernamentales, los propietarios de fábrica y los gerentes de nivel medio. También se llevó a cabo grupos de discusión con los trabajadores de las fábricas. Así, trató de entender las nociones básicas de cada grupo sobre la competitividad y lo que veían como factores clave a nivel de fábrica y en el plano nacional. Aunque hubo desacuerdos entre estos diferentes grupos sobre diversos temas, la productividad fue identificada principalmente como el factor más importante para garantizar la competitividad de Bangladesh.

[8] representan la relación entre los diversos grupos y la influencia de factores externos sobre las reformas, tanto a nivel nacional como de fábrica (Tabla 5). Realizan un estudio de factores por cada grupo de interés, para ello desarrollaron tablas en las que se considera a cada uno de los factores principales; así, presentan los factores que son relevantes por tres grupos de interés: los trabajadores de las fábricas, los dueños de las fábricas y los compradores internacionales.

Tabla 5: Factores que influyen en la competitividad de las fábricas a finales del 2008 [8]

\begin{tabular}{|c|c|c|}
\hline $\begin{array}{c}\text { Trabajadores de } \\
\text { fabrica }\end{array}$ & $\begin{array}{c}\text { Propietarios de la } \\
\text { fábrica }\end{array}$ & $\begin{array}{c}\text { Minorista } \\
\text { internacional }\end{array}$ \\
\hline Calidad del producto & Velocidad al mercado & $\begin{array}{l}\text { Bajo costo / Calidad } \\
\text { / Confiabilidad / } \\
\text { Pre-producción de } \\
\text { asistencia }\end{array}$ \\
\hline Salario & $\begin{array}{l}\text { Productividad de los } \\
\text { trabajadores }\end{array}$ & $\begin{array}{l}\text { Velocidad al mercado } \\
\text { / Normas laborales }\end{array}$ \\
\hline $\begin{array}{l}\text { Buenas condiciones } \\
\text { de trabajo / Cuidado } \\
\text { de la Salud }\end{array}$ & $\begin{array}{l}\text { Buenas condiciones de } \\
\text { trabajo / Salud }\end{array}$ & $\begin{array}{l}\text { Capacidad para mane- } \\
\text { jar diseños y materia- } \\
\text { les sofisticados }\end{array}$ \\
\hline \multirow[t]{6}{*}{ Formación } & $\begin{array}{l}\text { Relación previa de largo } \\
\text { plazo con su comprador }\end{array}$ & \\
\hline & $\begin{array}{l}\text { Salarios dignos de los } \\
\text { trabajadores }\end{array}$ & \\
\hline & Avances tecnológicos & \\
\hline & $\begin{array}{l}\text { Asistencia / capacidad } \\
\text { de pre-producción para } \\
\text { manejar diseños / ma- } \\
\text { teriales sofisticados }\end{array}$ & \\
\hline & $\begin{array}{l}\text { Formación de los traba- } \\
\text { jadores }\end{array}$ & \\
\hline & Bajo costo & \\
\hline
\end{tabular}




\section{Modelo propuesto}

Tomando en cuenta que la aplicación del Análisis Envolvente de Datos (DEA) para medir la eficiencia en diferentes escenarios y sectores ha sido comprobada en su totalidad [30], [31], [32], [33], [34], se aplicará DEA para la evaluación de la competitividad de las empresas de manufactura textil. Esta medición se realiza con base en la diversidad de variables relacionadas al sector.

Según Mauricio [12], para que las industrias y las empresas de servicios aumenten su nivel de competitividad, es necesario que estas realicen acciones orientadas a mejorar la calidad, eficiencia e innovación. Es decir la competitividad es influenciada por los factores Calidad, Innovación y Eficiencia. A su vez, el Reporte Financiero Burkenroad Perú - Sector Textil del Perú, 2009 señala: “...en ventajas competitivas entre las que podemos mencionar la alta calidad y el prestigio de las fibras peruanas..." [36], confirmando la influencia del factor Calidad en la competitividad.

El modelo propuesto se divide en dos fases (ver Fig. 2). En la primera fase, se realiza una medición de tres factores (Calidad, Innovación y Eficiencia), considerando las variables de cada factor. Los resultados de la primera fase se convierten en las entradas de la segunda fase. En la segunda fase, se mide la competitividad considerando los tres factores obtenidos en la fase uno. De esta manera se obtienen los resultados para definir la frontera de Pareto que permite identificar el nivel de competitividad de cada empresa evaluada. Así mismo, la primera evaluación permitirá conocer el nivel de desarrollo en cada uno de los factores.

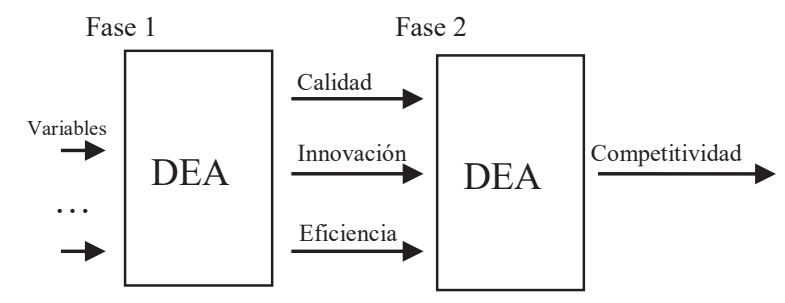

Fig. 2: Modelo basado en DEA para medir la competitividad de las manufactureras peruanas del sector textil.

\subsection{Fase1: Evaluación de Calidad, Innovación, Eficiencia}

En la primera fase del modelo, para medir los factores Calidad, Innovación y Eficiencia se han identificado de la literatura concerniente al sector textil y a otros sectores industriales las variables que influyen en la $\mathrm{Ca}-$ lidad, Innovación y Eficiencia (ver respectivamente las tablas 6,7 y 8 ).

De la Tabla 6, las variables Diseño de alta calidad, Alta calidad de los productos, Calidad del trabajo y Calidad de los proveedores locales influyen en la Calidad pero no pertenecen al sector textil, por lo que estas serán consideradas en el presente estudio
El modelo DEA para medir el factor de Calidad, es dado como sigue:

$$
\begin{aligned}
& \text { Maximizar } \beta=\frac{u_{1} y_{1}}{\sum_{j=1}^{m} v_{j} x_{1 j}} \\
& \text { Sujeto a }: 0 \geq \frac{u_{1} y_{1}}{\sum_{j=1}^{m} v_{j} x_{1 j}} \leq 1 \\
& \quad u_{1}, v_{i} \geq 0
\end{aligned}
$$

Donde:

$\boldsymbol{u}_{\mathbf{1}}$ es el peso determinado para la Calidad

$v_{\boldsymbol{i}}$ es el peso determinado para la entrada $x_{1 i}$

$\boldsymbol{m}$ es el número de entradas $=14$

$\boldsymbol{\beta}$ es la Calidad relativa de cada organización.

La DMUj consume la cantidad $x_{1 j}$ de la entrada i y produce la Calidad $y_{1}$. Para medir el nivel de desarrollo de la Calidad de la DMUo, se resuelve un problema de optimización que busca maximizar la razón de sus salidas entre sus entradas. Ninguna DMU puede tener una ponderación de Calidad mayor al 100\%.

Tabla 6: Lista de variables para el factor Calidad

\begin{tabular}{|c|l|c|}
\hline \multicolumn{1}{|c|}{ Id } & \multicolumn{1}{|c|}{ Variable } & Referencia \\
\hline$x_{1,1}$ & Diseño de alta calidad & {$[37]$} \\
\hline$x_{1,2}$ & Calidad del producto & {$[6]$} \\
\hline$x_{1,3}$ & Servicio al cliente & {$[6]$} \\
\hline$x_{1,4}$ & Calidad & {$[6][9]$} \\
\hline$x_{1,5}$ & Materias primas & {$[9]$} \\
\hline$x_{1,6}$ & Calidad de demanda & {$[9]$} \\
\hline$x_{1,7}$ & Calidad de proveedores locales & {$[9]$} \\
\hline$x_{1,8}$ & Estructura y rivalidad (diferenciación) & {$[9]$} \\
\hline$x_{1,9}$ & Alta calidad de los productos & {$[23]$} \\
\hline$x_{1,10}$ & Calidad del trabajo & {$[23]$} \\
\hline$x_{1,11}$ & Demanda del consumidor (local y extranjero) & {$[35]$} \\
\hline$x_{1,12}$ & Calidad (ecología textil) & {$[35]$} \\
\hline$x_{1,13}$ & Satisfacción del cliente & {$[35]$} \\
\hline$x_{1,14}$ & Calidad de los proveedores locales & {$[25]$} \\
\hline
\end{tabular}

De la Tabla 7, las variables Aplicación de EDI (Encuentro de Diseño e Innovación) y Capacidad para la innovación no pertenecen al sector textil, pero tienen influencia sobre el factor Innovación, por lo que se han tomado en cuenta en la evaluación. 
El modelo DEA para medir el factor de Innovación, es dado como sigue:

$$
\begin{aligned}
& \text { Maximizar } \beta=\frac{u_{2} y_{2}}{\sum_{i=1}^{m} v_{j} x_{2 j}} \\
& \text { Sujeto a }: 0 \geq \frac{u_{2} y_{2}}{\sum_{j=1}^{m} v_{j} x_{2 j}} \leq 1 \\
& u_{2}, v_{i} \geq 0
\end{aligned}
$$

Donde:

$\boldsymbol{u}_{\boldsymbol{r}}$ es el peso determinado para la Innovación

$\boldsymbol{v}_{\boldsymbol{i}}$ es el peso determinado para la entrada $x_{2 i}$

$\boldsymbol{m}$ es el número de entradas $=12$

$\boldsymbol{\beta}$ es la Innovación relativa de cada organización.

La DMUj consume la cantidad $x_{2 j}$ de la entrada i y produce la Innovación $y_{2}$. Para medir el nivel de desarrollo de la Innovación de la DMUo, se resuelve un problema de optimización que busca maximizar la razón de sus salidas entre sus entradas. Ninguna DMU puede tener una ponderación de Calidad mayor al 100\%.

Tabla 7: Lista de variables para el factor Innovación

\begin{tabular}{|c|l|c|}
\hline Id & \multicolumn{1}{|c|}{ Variable } & Referencia \\
\hline$x_{2,1}$ & $\begin{array}{l}\text { Aplicación de EDI(Encuentro de Diseño e } \\
\text { Innovación) }\end{array}$ & {$[37]$} \\
\hline$x_{2,2}$ & $\begin{array}{l}\text { Innovación en términos de desarrollo de la } \\
\text { producción }\end{array}$ & {$[6]$} \\
\hline$x_{2,3}$ & Innovación y desarrollo & {$[6]$} \\
\hline$x_{2,4}$ & Tecnología & {$[9]$} \\
\hline$x_{2,5}$ & Cooperación universidades con la industria & {$[9]$} \\
\hline$x_{2,6}$ & Posibilidad de difusión de la tecnología & {$[9]$} \\
\hline$x_{2,7}$ & Soporte de los procesos tecnológicos & {$[9]$} \\
\hline$x_{2,8}$ & Innovación y desarrollo tecnológico & {$[35]$} \\
\hline$x_{2,9}$ & Maximizar la productividad & {$[35]$} \\
\hline$x_{2,10}$ & Innovación (eco sellos) & {$[35]$} \\
\hline$x_{2,11}$ & $\begin{array}{l}\text { Estrategia (crear diseños y tendencias } \\
\text { forjando creatividad) }\end{array}$ & {$[35]$} \\
\hline$x_{2,12}$ & Capacidad para la innovación & {$[25]$} \\
\hline
\end{tabular}

De la Tabla 8, las variables Sofisticación en el proceso de producción, Agilidad, Habilidades de alta producción, Mano de Obra calificada, Calidad en la educación, Inversión en investigación y desarrollo e Infraestructura logística y administrativa no pertenecen al sector textil, pero tienen influencia sobre el factor Innovación, por lo que se han tomado en cuenta en la evaluación.
El modelo DEA para medir el factor de Eficiencia, es dado como sigue:

$$
\begin{aligned}
& \text { Maximizar } \beta=\frac{u_{3} y_{3}}{\sum_{j=1}^{m} v_{j} x_{3 j}} \\
& \text { Sujeto a : } 0 \geq \frac{u_{3} y_{3}}{\sum_{j=1}^{m} v_{j} x_{3 j}} \leq 1
\end{aligned}
$$$$
u_{3}, v_{i} \geq 0
$$

Donde:

$\boldsymbol{u}_{\boldsymbol{r}}$ es el peso determinado para la Eficiencia

$\boldsymbol{v}_{\boldsymbol{i}}$ es el peso determinado para la entrada $x_{3 i}$

$\boldsymbol{m}$ es el número de entradas $=17$

$\boldsymbol{\beta}$ es la Eficiencia relativa de cada organización.

La DMUj consume la cantidad $x_{3 j}$ de la entrada i y produce la Eficiencia $y_{3}$. Para medir el nivel de desarrollo de la Eficiencia de la DMUo, se resuelve un problema de optimización que busca maximizar la razón de sus salidas entre sus entradas. Ninguna DMU puede tener una ponderación de Calidad mayor al $100 \%$.

Tabla 8: Lista de variables para el factor Eficiencia

\begin{tabular}{|c|l|c|}
\hline Factor & \multicolumn{1}{|c|}{ Variable } & Referencia \\
\hline$x_{3,1}$ & Sofisticación en el proceso de producción & {$[37]$} \\
\hline$x_{3,2}$ & Agilidad & {$[37]$} \\
\hline$x_{3,3}$ & Integración vertical & {$[6]$} \\
\hline$x_{3,4}$ & Flexibilidad & {$[6]$} \\
\hline$x_{3,5}$ & Autonomía de los empleados & {$[6]$} \\
\hline$x_{3,6}$ & Bajo inventario & {$[6]$} \\
\hline$x_{3,7}$ & Control de la cadena de suministro & {$[6]$} \\
\hline$x_{3,8}$ & Recursos Humanos especializado & {$[25]$} \\
\hline$x_{3,9}$ & $\begin{array}{l}\text { Infraestructura física (vías, electricidad, } \\
\text { puertos) }\end{array}$ & {$[3]$} \\
\hline$x_{3,10}$ & Habilidades de alta producción & {$[3]$} \\
\hline$x_{3,11}$ & Mano de Obra calificada & {$[35]$} \\
\hline$x_{3,12}$ & Mano de Obra calificada & {$[25]$} \\
\hline$x_{3,13}$ & $\begin{array}{l}\text { Infraestructura (Capacidad productiva, acceso } \\
\text { a la tecnología) }\end{array}$ \\
\hline$x_{3,14}$ & Capacitación del talento humano tecnológica & {$[35]$} \\
\hline$x_{3,15}$ & Calidad en la educación & [23] \\
\hline$x_{3,16}$ & Inversión en investigación y desarrollo & {$[3$ fraestructura logística y administrativa } \\
\hline
\end{tabular}




\subsection{Evaluación de la competitividad}

El modelo DEA para medir la competitividad, es dado como sigue:

$$
\begin{aligned}
& \text { Maximizar } \beta=\frac{\sum_{r=1}^{s} u_{r} y}{\sum_{i=1}^{m} v_{i} x} \\
& \text { Sujeto a : } 0 \geq \frac{\sum_{r=1}^{s} u_{r} y}{\sum_{i=1}^{m} v_{i} x} \leq 1
\end{aligned}
$$$$
u_{r}, v_{i} \geq 0
$$

Donde:

$\boldsymbol{u}_{\boldsymbol{r}}$ es el peso determinado para la salida $\mathrm{r}$

$\boldsymbol{v}_{\boldsymbol{i}}$ es el peso determinado para la entrada i

$\boldsymbol{s}$ es el número salidas $=1$

$\boldsymbol{m}$ es el número de entradas $=3$

$\boldsymbol{\beta}$ es la Competitividad relativa de cada empresa.

Es la competitividad relativa de cada organización.

La aplicación del análisis envolvente de datos tiene como objetivo el resolver, de manera exitosa, problemas en los que la diversificación de los factores a evaluar, de manera cuantitativa, generan un entorno polémico como lo es este tema en particular "medir la competitividad".

Por lo que al aplicar DEA se puede destacar que logramos obtener las siguientes ventajas:

- Evita la arbitrariedad [33]

- Genera un área de factibilidad técnica

- Se habla de dominancia

- Se realiza un benchmarking[33]

- DEA no requiere una hipótesis de relación funcional entre dichos inputs y outputs.

- Las unidades se comparan directamente con otras unidades o una combinación de ellas.

Así mismo, hay ciertas reglas que cumplir, las cuales se presentan a continuación como desventajas:

- Dado que DEA es una técnica de punto extremo, ruidos (incluso las distorsiones simétricas con media cero), tales como errores de medición, pueden causar problemas significativos.

- Los test de hipótesis estadísticas son difíciles de aplicar, por ser un método no paramétrico.

- Este tipo de análisis funciona relativamente mal cuando el número de DMU es bajo.
Las unidades se comparan directamente con otras unidades o una combinación de ellas.

\section{Resultados}

Para efectos de mostrar el uso del modelo se han utilizado 16 empresas manufactureras ( Tabla 9 ) cuyos datos se encuentran en la Encuesta Nacional de Innovación en la Industria Manufacturera del año 2015 [40] y la Estadística Industrial Mensual del 2012 en el Perú.

Tabla 9: Listado de empresas evaluadas

\begin{tabular}{|c|l|}
\hline Id & Información de la empresa \\
\hline A & Aris Industrial SA \\
\hline B & Compañia Industrial Nuevo Mundo SA \\
\hline C & Creditex S.A.A. \\
\hline D & Compañia Universal Textil \\
\hline E & Confecciones Lancaster \\
\hline F & Consorcio la Parcela \\
\hline G & Filasur SA \\
\hline H & Hilanderia de Algodón Peruano SA \\
\hline I & Inca Tops SA \\
\hline J & Tecnología Textil SA \\
\hline K & Tejidos San Jacinto SA \\
\hline L & Texfina SA \\
\hline M & Textil El Amazonas SA \\
\hline N & Textil Oceano SAC \\
\hline O & Textil San Ramon \\
\hline P & Textiles Bustamante SA \\
\hline
\end{tabular}

\subsection{Resultados sobre Calidad, Innovación, Eficiencia}

Se aplicó el software SIAD para evaluar los factores calidad, innovación y eficiencia según los modelos DEA propuestos.

En la Fig. 4, se muestra el ranking de las manufactureras del sector textil con respecto al factor calidad, donde claramente se puede apreciar que la manufacturera "G" es la más eficiente, seguida de la manufacturera "D" y la manufacturera "K".

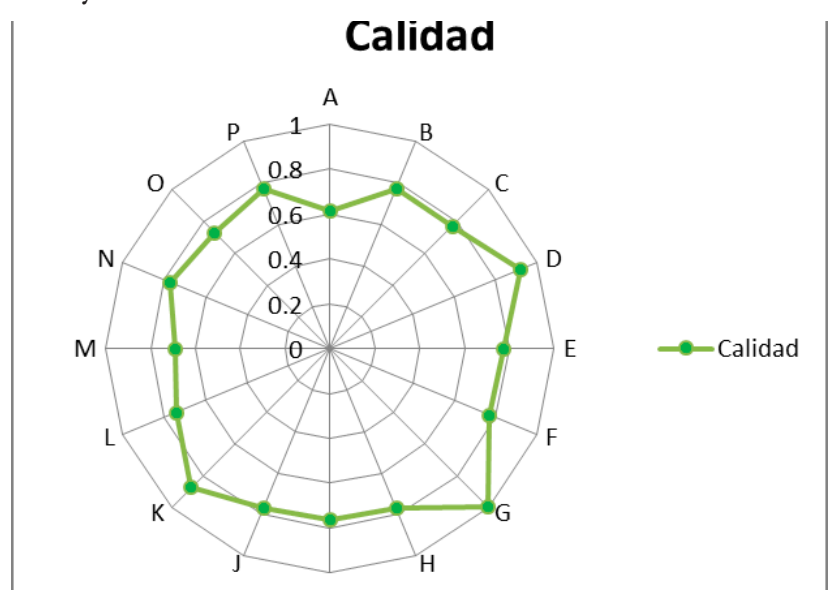

Fig. 4: Evaluación de la Calidad con el modelo aplicando DEA para medir la competitividad de las Manufactureras del sector Textil 
En la Fig. 5, se muestra el ranking de las manufactureras del sector textil con respecto al factor innovación, donde se puede apreciar que las manufactureras "B", "G" y "I" son las unidades evaluadas que formarán la frontera de Pareto, es decir, son las más eficientes, en relación con la muestra evaluada.

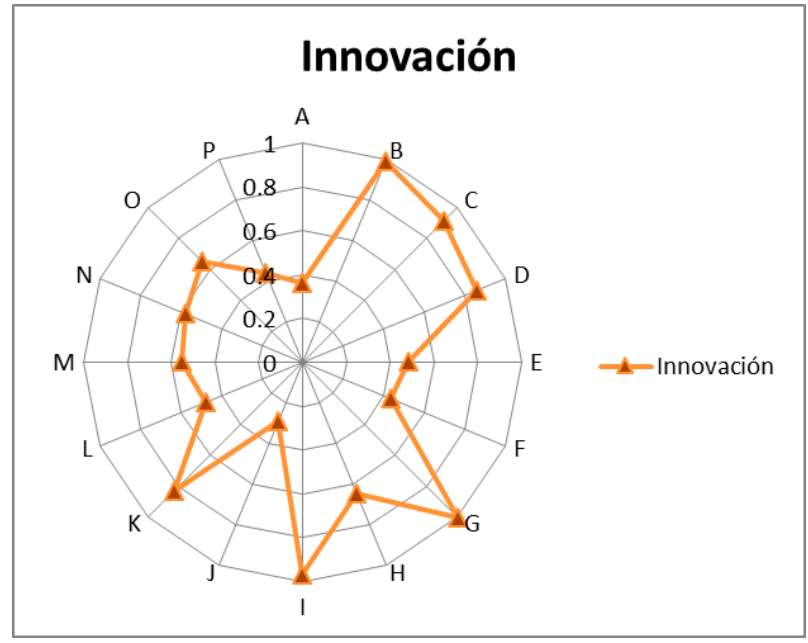

Fig. 5: Evaluación de la Innovación con el modelo aplicando DEA para medir la competitividad de las Manufactureras del sector Textil

En la Fig. 6, se muestra el ranking de las manufactureras del sector textil con respecto al factor eficiencia, donde se puede apreciar que las manufactureras "M", "O" y "B" son las unidades evaluadas que formaran la frontera de Pareto, es decir, son las más eficientes, en relación con la muestra evaluada.

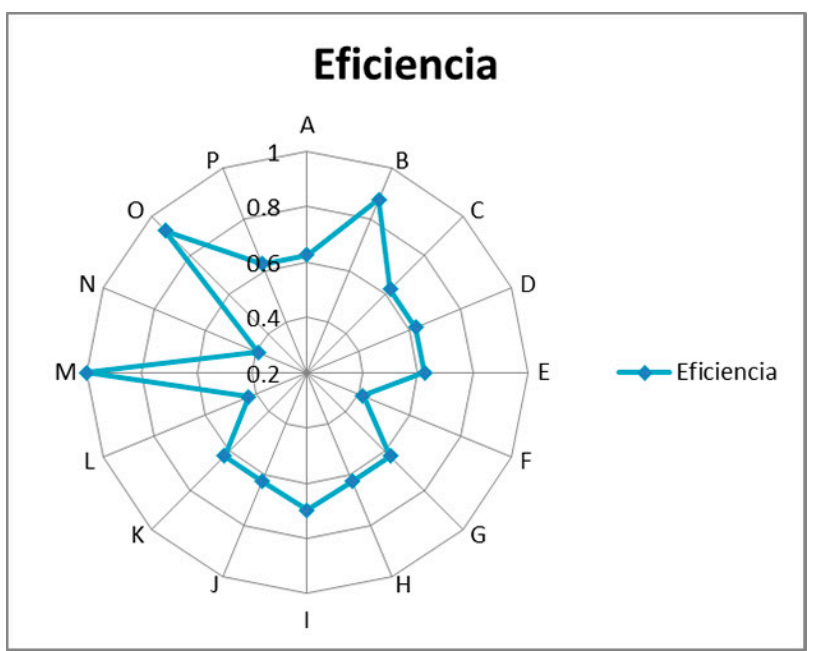

Fig. 6: Evaluación de la Eficiencia con el modelo aplicando DEA para medir la competitividad de las Manufactureras del sector Textil

\subsection{Resultados sobre Competitividad}

Se aplicó el software SIAD para evaluar La competitividad según el modelo DEA propuesto.

En la Fig. 7, se muestra los tres factores calidad, innovación y eficiencia con cada uno de los valores obtenidos en las evaluaciones para cada una de las manufactureras del sector textil (representadas en letras desde la A hasta la P). La evaluación de los tres factores da como resultado la evaluación de la competitividad en el sector textil, representado en la Fig. 7.

Como se observa en la Fig. 7, hay manufactureras que destacan en alguno de los factores; sin embargo, la evaluación está relacionada a las variables que conforman los factores definidos.

Cada uno de los valores obtenidos por factor en las evaluaciones para las manufactureras del sector textil da como resultado la evaluación de la competitividad en el sector textil, representado en la Fig. 7.

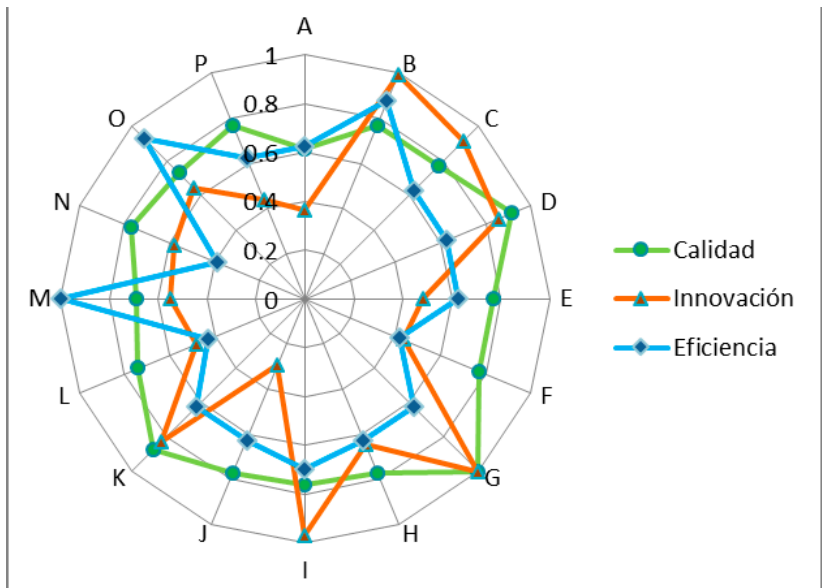

Fig. 7: Evaluación de la Competitividad con el modelo basado en DEA para medir la competitividad de las Manufactureras del sector Textil

En la Fig. 8, se muestra el ranking de competitividad de las manufactureras del sector textil, donde se puede apreciar que las manufactureras "M", "O", "A" y "J" son las unidades evaluadas que formarán la frontera de Pareto, es decir, son las más eficientes, en relación a los tres factores evaluados conformados por calidad, innovación y eficiencia.

\section{Competitividad}

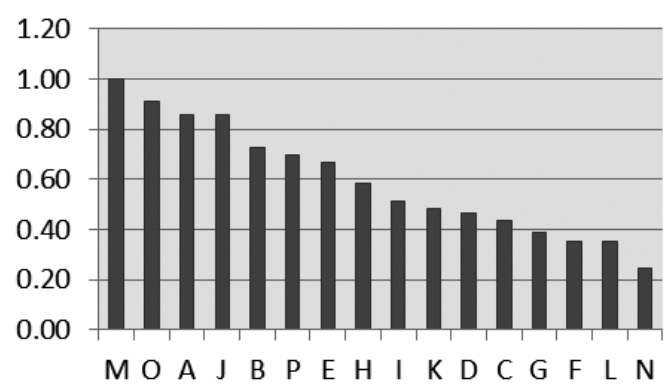

Fig. 8: Evaluación de la Competitividad con el modelo basado en DEA para medir la competitividad de las Manufactureras del sector Textil

De los resultados obtenidos y que se presentan gráficamente, se concluye que la aplicación de DEA para medir la competitividad es factible porque identifica la competitividad de las empresas evaluadas, indica el nivel de acuerdo a una escala (la ubicación) de cada empresa basado en la frontera de Pareto, identifica las empresas que se encuentran en la frontera de Pareto y permite 
identificar los factores en los que deben tomar acciones para obtener mejores resultados con base en la realidad de cada empresa.

\section{Conclusiones}

Se diseñó un modelo aplicando DEA para medir la competitividad de las manufactureras peruanas del sector textil. El modelo cuenta con variables de entrada definidas. Se explica la aplicación de DEA para la evaluación de los datos y el análisis de los resultados.

Se definió como los factores del modelo para medir la competitividad en las manufactureras peruanas: Calidad, Eficiencia e Innovación. Dos de los factores (Eficiencia e Innovación) son considerados indispensables para la medición de competitividad para el WEF, que en las tres divisiones de sus 12 pilares identifica los factores indicados, por lo que se tomó como referencia y como parte del aporte se agregó el factor Calidad (factor de diferenciación de acuerdo a la literatura revisada) para la medición de la competitividad en las manufactureras peruanas.

Esta investigación tiene como alcance el sector manufacturero textil peruano. Para evaluar la competitividad de este sector, es necesario comprometer la participación de los interesados, mantener la continuidad de esta evaluación de modo que con la continuidad este modelo pueda nutrirse y ampliar los factores propuestos. El modelo propone inicialmente tres factores: la calidad, la eficiencia y la innovación.

De los resultados obtenidos, se concluye que la aplicación de DEA para medir la competitividad es factible, porque identifica la competitividad de las empresas evaluadas, indica el nivel de acuerdo a una escala (la ubicación) de cada empresa basado en la frontera de Pareto, identifica las empresas que se encuentran en la frontera de Pareto y permite identificar las variables en los que deben tomar acciones para obtener mejores resultados con base en la realidad de cada empresa.

La aplicación del modelo en otros escenarios no tiene limitantes, sin embargo, se recomienda realizar una evaluación para comprobar si los factores propuestos para el sector manufacturero textil peruano son suficientes para la aplicación del modelo en otros sectores o es necesario agregar más factores antes de su aplicación.

\section{Agradecimientos}

Agradezco al Dr. David Mauricio Sánchez por sus comentarios y sugerencias a la presente investigación.

\section{Referencias}

[1] Porter, M. E. (1990). The competitive advantage of notions. Harvard Business Review.

[2] Kudrle, R. T. (1994). Three perspectives on competitiveness: an introduction to "Made in America." The International Executive, 38(4), 403-429.
[3] Jinbei. (2003). Economics of Competitiveness. Guangzhou: Guangdong Economy Press. No.4.

[4] Chan, S. Y., y Suk-Yee Lee, D. (2003). An empirical investigation of symptoms of obsolete costing systems and overhead cost structure. Managerial Auditing Journal, 18(2), 81-89.

[5] Triveño, G. (23 de septiembre de 2017). A propósito del sector textil confecciones. Gestion.pe. Recuperado de https:// gestion.pe/blog/prosperoperu/2017/10/a-proposito-del-sector-textil-confecciones.html.

[6] Li, H., Li, V., Skitmore, M., Wong, J. K. W., y Cheng, E. W. L. (2009). Competitiveness factors: a study of the real estate market in China. Construction Management and Economics, 27(6), 567-579. https://doi.org/10.1080/01446190903005865

[7] Rojaka, J. (2009). Baltic states' competitiveness: before and after the global crisis. Taikomoji ekonomika: sisteminiai tyrimai, 2009, p. 27-46.

[8] Saxena, S. B., y Salze-Lozac'h, V. (2010). Competitiveness in the Garment and Textiles Industry: Creating a supportive environment. A Case Study of Bangladesh.

[9] Shafaei, R. (2009). An analytical approach to assessing the competitiveness in the textile industry. Journal of Fashion Marketing and Management: An International Journal, 13(1), 20-36.

[10] Ajitabh, A. and S. Momaya, K. (2003). Competitiveness of Firms: Review of Theory, Frameworks and Models. Singapore Management Review, 26(1), pp. 45-61.

[11] Schuller, B. J., y Lidbom, M. (2009). Competitiveness of Nations in the Global Economy. Is Europe Internationally Competitive? Economics and Management, (14), 934-939.

[12] Mauricio S. (2001), La competitividad en la industria y la ingeniería de sistemas. Industrial data (4) 1: 61-65. http://revistasinvestigacion.unmsm.edu.pe/index.php/idata/article/ view/6602/5885

[13] Parrish, Erin and Berdine, Matt and Cassill, Nancy and Oxenham, William, Measuring the Competitive Advantage of the US Textile and Apparel Industry (2008). 2008 Industry Studies Conference Paper. Available at SSRN: https://ssrn.com/ abstract=1134985 or http://dx.doi.org/10.2139/ssrn.1134985

[14] Drew, D., y Skitmore, M. (1997). The effect of contract type and size on competitiveness in bidding. Construction Management \& Economics, 15(5), 469-489.

[15] Cartwright, W. R. (1993). Multiple linked "diamonds" and the international competitiveness of export-dependent industries: The New Zealand experience. MIR: Management International Review, 55-70.

[16] Cho, D. y Moon, H. (2000). From Adam Smith to Michael Porter: Evolution of Competitiveness Theory. Korea: Asia-Pacific Business Series, p. 223.

[17] Hamalainen, T. J. (2003). National competitiveness and economic growth. Gloucestershire: Edward Elgar Publishing Limited.

[18] Zanakis, S. H., y Becerra-Fernandez, I. (2005). Competitiveness of nations: A knowledge discovery examination. European Journal of Operational Research, 166(1), 185-211.

[19] WEF (World Economic Forum) (2012). The global competitiveness report 2012-2013. Switzerland. 
[20] Oral, M., y Reisman, A. (1988). Measuring industrial competitiveness. Industrial Marketing Management, 17(3), 263-272.

[21] Guan, J. C., Yam, R. C., Mok, C. K., y Ma, N. (2006). A study of the relationship between competitiveness and technological innovation capability based on DEA models. European Journal of Operational Research, 170(3), 971-986.

[22] Simona, G.-L., y Axèle, G. (2012). Knowledge transfer from TNCs and upgrading of domestic firms: the Polish automotive sector. World Development, 40(4), 796-807.

[23] Yanrong, W., Yu, L., y Kang, L. (2011). Evaluation on the Competitiveness of High-tech Entrepreneurial Enterprises. Energy Procedia, 5, 684-689.

[24] Cinicioglu, E. N., Önsel, Ş., y Ülengin, F. (2012). Competitiveness analysis of automotive industry in Turkey using Bayesian networks. Expert Systems with Applications, 39(12), 10923-10932.

[25] Mitschke, A. (2008). The Influence of National Competition Policy on the International Competitiveness of Nations: $A$ Contribution to the Debate on International Competition Rules. Springer Science \& Business Media.

[26] Balassa, B. (1965). Trade Liberalisation and "Revealed" Comparative Advantage1. The Manchester School, 33(2), 99-123. https://doi.org/10.1111/j.1467-9957.1965.tb00050.x

[27] Dobrescu, E. (2010). Macromodel Simulations for the Romanian Economy (SSRN Scholarly Paper No. ID 1620521). Rochester, NY: Social Science Research Network.

[28] Zhang, L., Wei, H., Li, Z., Zhang, Y., Liu, Y., Lu, S., y Zhang, P. (2013). Intelligent and Integrated Sustainable Multimodal Transportation Systems Proceedings from the 13th COTA International Conference of Transportation Professionals (CICTP2013) Port Competitiveness Evaluation Research based on Chernooff Faces Model. Procedia - Social and Behavioral Sciences, 96, 1961-1966. https://doi.org/10.1016/j. sbspro.2013.08.221

[29] Hoefter, A. F. (2001). The competitiveness of Ghana's industry (Doctoral dissertation, Difo-Druck).

[30] Fernández, M. del P. M. (2004). Eficiencia técnica y calidad del output en la Universidad de Santiago de Compostela. ResearchGate, 5(1). Retrieved from https://www.researchgate.net/publication/26423620_Eficiencia_tecnica_y_calidad_del_output_en_la_Universidad_de_Santiago_de_Compostela
[31] Mejía, J. A. S., Valencia, W. A., \& Carpintero, Á. A. T. (2005). La producción académica como uno de los indicadores del desempeño de las universidades públicas colombianas desde la perspectiva del análisis envolvente de datos. Scientia et Technica, 2(28), 109-114.

[32] Silva Santiago, C. V., Santiago, C. V. S., \& Arellano, A. R. de. (2006). Análisis de Eficiencia de Institutos Tecnológicos de España y Brasil: Una Aplicación del Análisis Envolvente de Datos (DEA). Journal of Technology Management \& Innovation, 1(4), 43-56.

[33] Charles, V., \& Zegarra, L. F. (2014). Measuring regional competitiveness through Data Envelopment Analysis: A Peruvian case. Expert Systems with Applications, 41(11), 5371-5381. http://doi.org/10.1016/j.eswa.2014.03.003

[34] Herrera, T. F., Mendoza, A. M., \& Cadavid, D. V. (2015). Análisis comparativo de eficiencia financiera: Estudio de un caso sectorial en Barranquilla. Prospectiva, 13(2), 16-24. http:// doi.org/10.15665/rp.v13i2.483

[35] Alarcón Reyes, N. Y. (2011). Análisis del sector textil-confección colombiano, sus estrategias de competitividad en el período 2000-2009 y la aplicabilidad del modelo INEXMODA en Ecuador. Retrieved from http://repositorio.puce.edu.ec/ handle/22000/3871

[36] Cerrato, D. y Depperu, D. (2011). Unbundling the construct of firm level international competitiveness. Multinational Business Review, 19(4), 311-331. https://doi. org/10.1108/15253831111190162

[37] Messner, D., y Meyer-Stamer, J. (1994). Systemic competitiveness: Lessons from Latin America and beyond - perspectives for Eastern Europe. The European Journal of Development Research, 6(1), 89-107. https://doi. org/10.1080/09578819408426599

[38] Farrell, M. J. (1957). The Measurement of Productive Efficiency, 120(3), 253-290.

[39] Charnes, A., Cooper, W. W., \& Rhodes, E. (1978). Measuring the efficiency of decision making units. European Journal of Operational Research, 2(6), 429-444. https://doi. org/10.1016/0377-2217(78)90138-8

[40] Manufactura 2007 - 2015. (n.d.). INEI. Retrieved from https:// www.inei.gob.pe/media/MenuRecursivo/indices_tematicos/ pbi_act04_1.xlsx 